\title{
Development of a fluorescence-based multiplex genotyping method for simultaneous determination of human papillomavirus infections and viral loads
}

\author{
Zhengrong Sun ${ }^{1+}$, Rong Zhang ${ }^{2 \dagger}$, Zhonghua Liu², Chao Liu', Xiulin Li ${ }^{2}$, Weiqiang Zhou', Lianxia Yang ${ }^{1}$, \\ Qiang Ruan ${ }^{1 *}$ and Xu Zhang ${ }^{2 *}$
}

\begin{abstract}
Background: Persistent high-risk human papillomavirus (HPV) infection is correlated with an increased risk of developing intraepithelial lesion or malignancy (NILM). The aims of the current study is to establish a method named BioPerfectus Multiplex Real Time (BMRT) HPV assay for simultaneous typing and quantifying HPVs, and to evaluate it by comparison with HPV GenoArray test and PCR-sequencing method, as well as histological status.

Methods: A total of 817 cervical specimens were evaluated by BMRT method and HPV GenoArray test, using PCRsequencing method as the reference standard; simultaneously, high-risk HPV-16 and -18 DNA loads were assessed in 443 specimens to investigate the correlation with infection outcomes.

Results: The overall detection coincidence rate between BMRT assay and HPV GenoArray test is $96.6 \%$ and the Kappa value is 0.760 . In addition, the sensitivity and positive predictive value of BMRT is $98.4 \%$ and $95.7 \%$ compared with the results detected by PCR-sequencing method, respectively. HPV-16 viral load has a correlation with CINs or worse lesions. By comparing with infected women presenting NILM /cervicitis, the cutoff value for HPV-16 from patients with CINs was 0.827 . With this cutoff value, $74.6 \%$ sensitivity and $72.5 \%$ specificity for prediction of HPV-16 infected patients with $\mathrm{CINI}$ and higher CIN were achieved. High significance was obtained when comparing the infected women presenting NILM/cervicitis with women either with CIN and cervical carcinomas $(p<0.001)$.
\end{abstract}

Conclusions: The BMRT assay seemed to be a good alternative approach for HR-HPV testing, due to its high level of automation and ability to quantify HPV-16, HPV-18 and other HR-HPVs.

Keywords: HPV, BMRT, GenoArray test, Sequence, Cervical lesion

\section{Background}

Development of cervical cancer is usually related to an infection with human papillomavirus (HPV), especially with any of the 12 high-risk genotypes (HR, HPV-16, -18, -31, $-33,-35,-39,-45,-51,-52,-56,-58$ and -59$)$ [1-4]. HPV-16 and HPV-18 are the most common genotypes found in more than $70 \%$ of cervical cancer patients, in

\footnotetext{
* Correspondence: ruanq@sj-hospital.org; xuzhang@s-sbio.com

${ }^{\dagger}$ Equal contributors

'Virus Laboratory, The Affiliated Shengjing Hospital, China Medical University, Shenyang, Liaoning 110004, China

${ }^{2}$ Jiangsu Bioperfectus Technologies Limited Company, Jiangsu 225300, China
}

which HPV-16 can be detected in more than $50 \%$ cases $[5,6]$. It seems that HPV-16 is not only more common, but also more oncogenic [7]. Co-infection with multiple HPV types is common $[8,9]$. Studies show a tendency of some genotypes to cluster, and some genotypes to be inversely associated [10-12]. The biological significance of the individual infection in a multiple infection is however, difficult to establish. But, there is an association between multiple infections and increased risk of neoplasia compared to single infections $[8,13,14]$.

HPV viral load, as a product of the number of infected cells and the number of virus per infected cell, is therefore influenced by two main factors: the extent of an 
HPV infection on the cervical surface and the level of viral production in the infection area. Viral load has been suggested to be a potential biomarker for cervical intraepithelial neoplasia grade II (CINII) or higher CIN. However, there is no consistent evidence that a one-time measurement of viral load is a useful marker of prevalent disease or disease progression so far [15]. The impact of viral load change has been assessed in only a few studies $[16,17]$. An investigation in a hospitalized population of HPV-16 positive and cytologically normal women demonstrated that an increased HPV-16 viral load measured at six month interval was associated with a progress of CINII/III+ in infected women, while decreased viral load over time was more likely to be found in women who remained cytologically normal [17]. Changes in viral load and the associations of the changes with disease risk may imply the complex interaction between HPV and human host, and potentially serve as an additional predictive marker for the outcomes of infection.

At present, the most commercially used method for HPV genotyping is the HPV GenoArray test (HPV GenoArray test kit; Hybribio Ltd, Hong Kong) in China. The method is based on reverse line blot technology (RLB), in which the PCR products are hybridized to HPV type-specific probes on a membrane. In our previously research, HPV GenoArray test have been performed concerning the prevalence and distribution of HPV genotypes in women with cervical lesions from Liaoning Province, China, but the main drawbacks of the assays are its high material cost and its time-consuming performance [18]. In addition, it is difficult to give a diagnosis for borderline cases due to the read-outs being based on direct visualization only. Moreover, quantifications of viral DNA in samples are unavailable by this technique. In the study, the BioPerfectus Multiplex Real Time (BMRT) HPV assay was developed to detect $18 \mathrm{HR}-\mathrm{HPV}$ types and 3 low risk (LR) HPV types as well as the viral loads simultaneously, and the clinical value of the BMRT assay was estimated in cervical specimens. The purpose of the present study was to validate the BMRT HPV assay developed for detection of $21 \mathrm{HPVs}$, including $18 \mathrm{HR}-\mathrm{HPV}$ types of HPV-16, -18, -26, -31, $-33,-35,-39,-45,-51,-52,-53,-56,-58,-59,-66$, $-68,-73,-82$ and 3 LR-HPV types of HPV-6, -11, -81, by comparing with HPV GenoArray test and to evaluate whether the measurements of HPV-16 and -18 viral loads had potential diagnostic utility by comparing with histological diagnosis.

\section{Methods}

\section{Clinical samples}

Informed consent was obtained from participation in the study and this study was approved by the ethics committee of Affiliated Shengjing Hospital of China Medical University. Specimens were obtained from patients in the Department of Obstetrics and Gynecology of the hospital, who subjected for a routine diagnosis for HPV infection, between July 2011 and November 2012. Clinical data of the patients were collected. For each patient, cervical cells were scraped from the ecto- and endocervix with a cytobrush. The cervical specimens were placed in the PreservCyt ${ }^{\bullet}$ LBC medium (Cytyc, Bedford, MA, USA) and transported to the laboratory, where they were kept at temperatures between $2{ }^{\circ} \mathrm{C}$ and $8{ }^{\circ} \mathrm{C}$ until performance with a routine HPV GenoArray test. A total of $817 \mathrm{HPV}$ positive cervical samples detected previously, including 467 single positive samples and 350 multiple positive samples, were selected for the present study. Among them, 364 samples were HPV - 16 positive, 142 samples were HPV - 18 positive and 311 samples were positive for other HPV types in the routine laboratory detections. All patients in this retrospective study had liquid based cytology test or colposcopy done at the time the cervical scrapes were taken. The median age of the studied populations was 39 years old (range 18-66 years old) at the time the cervical scrapes were collected.

\section{DNA preparations}

Total cellular DNA from the residual samples was extracted using QIAamp DNA mini kit (Qiagen, Hilden, Germany), according to the manufacturer's instructions. The concentration of DNA was determined in a spectrophotometer (DU 640, Beckman Coulter). Successful extraction of human genomic DNA was evaluated by amplifying a 258-base pair (bp) fragment of glyceraldehyde 3-phosphate dehydrogenase (GAPDH) gene using primers 5 '-AGAAGGCTGG GGCTCATTTG-3' (forward) and 5'-AGGGG CCATCC ACAGTCTTC-3' (reverse). The PCR reactions were carried out in a thermo- cycler under the following conditions: an initial $95{ }^{\circ} \mathrm{C}$ for $9 \mathrm{~min} ; 40$ cycles of $94{ }^{\circ} \mathrm{C}$ for $20 \mathrm{~s}, 55^{\circ} \mathrm{C}$ for $30 \mathrm{~s}, 72{ }^{\circ} \mathrm{C}$ for $30 \mathrm{~s}$; and a final extension at $72{ }^{\circ} \mathrm{C}$ for 5 min. In each PCR assay, negative and positive controls were included. Only DNA preparations, from which the GAPDH DNAs were successfully amplified, were used for further analyses.

\section{HPV GenoArray test}

HPV GenoArray test was performed using HybriMax Kit (Hybribio Limited Corp., China) according to the manufacturer's instructions. Briefly, HPV specific fragments in the DNA preparations were amplified by PCR, and genotyping for HPVs was done by flow-through hybridization to a gene chip as described previously [18]. The gene chip contains type specific oligonucleotides immobilized on a nylon membrane, including $13 \mathrm{HR}-\mathrm{HPV}$ of HPV-16, -18, -31, $-33,-35,-39,-45,-51,-52,-56,-58,-59$ and $-68,5$ LR-HPVs of HPV-6, $-11,-42,-43$ and -44 , and HPV-53, -66 and -CP8304, which are popular in the Chinese 
population. The final results were determined by direct visualization of colorimetric changes on the chip.

\section{BMRT HPV PCR assay}

In the BMRT HPV PCR assay, PCR primers and corresponding TaqMan probes were designed to detect each of the 21 most prevalent HPV types, including $18 \mathrm{HR}$ HPV genotypes of HPV-16, -18, -26, -31, -33, -35, $-39,-45,-51,-52,-53,-56,-58,-59,-66,-68,-73$ and -82 , and 3 LR-HPV genotypes of HPV-6, -11 and -81 (equivalent to CP8304). A total of eight reactions per sample were performed simultaneously. Among them, the reactions A, B, C, D, E, F and G were prepared to simultaneously detect and differentiate HPV-16/-18/31, HPV-59/-66/-53, HPV-33/-58/-45, HPV-56/-52/-35, HPV-68/-51/-39, HPV-73/-26/-82 and HPV-6/-11/-81, respectively. Meanwhile, human TOP3, a single-copy gene encoding DNA topoisomerase III, was amplified in the reaction $\mathrm{H}$ as a control for determining relative number of viral copies in a given sample [19].

PCR amplification was conducted in a total reaction volume of $20 \mu \mathrm{L}$, which comprised $2 \mu \mathrm{L}$ DNA samples (up to 50 ng), $10 \mu \mathrm{L}$ Platinum Quantitative PCR SuperMix-UDG (Invitrogen), 10 pmol of each primer, and 1-5 pmol of

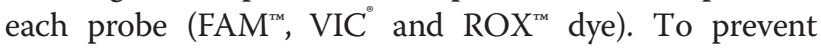
reamplification of carry-over PCR products, all reactions with Uracil-DNA-Glycosylase (UDG) were pre-incubated at $50{ }^{\circ} \mathrm{C}$ for $5 \mathrm{~min}$, followed by an initial denaturation at $95^{\circ} \mathrm{C}$ for $10 \mathrm{~min}$, which also inactivates UDG but activates the DNA polymerase, and 45 cycles at $95{ }^{\circ} \mathrm{C}$ for $10 \mathrm{~s}, 58{ }^{\circ} \mathrm{C}$ for 40 s. PCR was performed on an ABI Prism 7500 Detection System (Applied Biosystems).

Perfectus Software v1.0, which was used for genotyping and quantitative analysis of HPV nucleic acid (Bioperfectus Limited Corp., China), was applied for quantitative analyses of HPV-16 and -18 viral loads.

\section{Sequencing}

Products of HPV L1 gene amplified from samples by nested PCR using type-specific primers were purified with a QIAquick PCR Purification Kit (Qiagen, Hilden, Germany) as described by the manufacturer's instructions, and sequenced by Sangon Biotech Co., Ltd (Shanghai, China). Resulting DNA sequences were compared with the sequences of known HPV types using the basic local alignment search tool from the NCBI website (http://www.ncbi.nlm.nih.gov/BLAST).

\section{Statistical analysis}

Cohen's kappa value $(\mathrm{k})$ was calculated to assess the degree of agreement between results achieved by BMRT HPV PCR assay and HPV GenoArray test. Kappa values of $0-0.2,0.21-0.4,0.41-0.6,0.61-0.8,0.81-0.99$, and 1.0 indicate poor, slight, moderate, substantial, almost perfect and perfect agreement, respectively. $P$ values were calculated by Friedman Test. $P$ values $<0.05$ were considered to be statistically significant.

The accuracy measures of the BMRT HPV PCR assay for detecting $21 \mathrm{HPVs}$, including sensitivity, specificity, positive predictive value (PPV), negative predictive value (NPV) and their relative $95 \%$ confidence intervals (95\% CI) were determined according to sequencing results of PCR products.

Simultaneously, the accuracy measures for predicting CINs in HPV-16 infected patients by viral loads were stratified according to cytological and colposcopy grade. Four patient groups were set up, in which group 1 represents negative for intraepithelial lesion or malignancy (NILM), normal cytology and cervicitis; group 2 includes low-grade cervical intraepithelial neoplasia histology and observation of atypical squamous cells of undetermined significance (ASCUS) or low-grade squamous intraepithelial lesions (LSIL); group 3 is assigned for high-grade intraepithelial neoplasia or worse (CINII+) and high grade squamous intraepithelial lesions (HSIL); group 4 is for cancer. In order to calculate the prediction accuracy measures of BMRT HPV PCR assay for the cytology and colposcopy diagnoses, only HPV-16 positive cases in the four different thresholds were included. $P$ values were calculated by the Kruskal-Wallis test. For statistical analysis, the cytological and histological diagnosis was split between negative (CINI-III) and positive (cancer). Receiver operating characteristic (ROC) curve was constructed to find the clinical cutoff value, relative sensitivity and specificity of the BMRT HPV PCR assay. All statistical calculations were performed using the SPSS version 18.0 (SPSS Inc, Chicago, IL, USA).

\section{Results}

\section{Concordance rate of the BMRT HPV PCR assay with the} HPV GenoArray test

Results of infection status in both the multiple and single HPV positive samples were organized in a 2-by-2 crosstabulation for each HPV type, by classifying detection results of each sample as positive or negative for both of the BMRT HPV PCR assay and the HPV GenoArray test (Table 1). The overall HPV positive and negative coincidence rates between the two tests were $89.8 \%$ and $97.0 \%$, respectively; the total concordant coincidence was $96.6 \%$ yielding a kappa value of 0.760 . For detections of individual HPV types, the positive coincidence rate of the two methods was $100 \%$ for HPV-59, HPV-68 and HPV-51. An almost perfect agreement were obtained between the two methods for detection of HPV-16 $(k=0.844)$, HPV-18 $(k=0.881)$ and HPV-58 $(k=0.809)$, respectively. And a slight agreement was obtained for HPV-56, which showed the lowest kappa value of 0.284 . The discordant results were mainly caused by more HPV-positive samples 
Table 1 Concordance rate of the BMRT HPV PCR assay with the HPV GenoArray test

\begin{tabular}{|c|c|c|c|c|c|c|c|c|}
\hline Type $^{a}$ & $\begin{array}{l}\text { BMRT+/ } \\
\text { GenoArray+ }\end{array}$ & $\begin{array}{l}\text { BMRT+/ } \\
\text { GenoArray- }\end{array}$ & $\begin{array}{l}\text { BMRT-/ } \\
\text { GenoArray+ }\end{array}$ & $\begin{array}{l}\text { BMRT-/ } \\
\text { GenoArray- }\end{array}$ & $\begin{array}{l}\text { Positive } \\
\text { coincidence rate }\end{array}$ & $\begin{array}{l}\text { Negative } \\
\text { coincidence rate }\end{array}$ & Total coincidence rate & Kappa value \\
\hline HPV16 & 334 & 32 & 31 & 420 & $91.5 \%$ & $92.9 \%$ & $92.3 \%$ & 0.844 \\
\hline HPV18 & 129 & 10 & 18 & 660 & $87.8 \%$ & $98.5 \%$ & $96.6 \%$ & 0.881 \\
\hline HPV31 & 23 & 13 & 2 & 779 & $92.0 \%$ & $98.4 \%$ & $98.2 \%$ & 0.745 \\
\hline HPV59 & 16 & 19 & 0 & 782 & $100.0 \%$ & $97.6 \%$ & $97.7 \%$ & 0.617 \\
\hline HPV66 & 23 & 10 & 2 & 782 & $92.0 \%$ & $98.7 \%$ & $98.5 \%$ & 0.786 \\
\hline HPV53 & 37 & 20 & 2 & 758 & $94.9 \%$ & $97.4 \%$ & $97.3 \%$ & 0.757 \\
\hline HPV33 & 30 & 10 & 7 & 770 & $81.1 \%$ & $98.7 \%$ & $97.9 \%$ & 0.768 \\
\hline HPV58 & 73 & 13 & 17 & 714 & $81.1 \%$ & $98.2 \%$ & $96.3 \%$ & 0.809 \\
\hline HPV45 & 13 & 7 & 1 & 796 & $92.9 \%$ & $99.1 \%$ & $99.0 \%$ & 0.760 \\
\hline HPV56 & 12 & 53 & 2 & 750 & $85.7 \%$ & $93.4 \%$ & $93.3 \%$ & 0.284 \\
\hline HPV52 & 44 & 65 & 6 & 702 & $88.0 \%$ & $91.5 \%$ & $91.3 \%$ & 0.513 \\
\hline HPV35 & 9 & 15 & 1 & 792 & $90.0 \%$ & $98.1 \%$ & $98.0 \%$ & 0.521 \\
\hline HPV68 & 18 & 31 & 0 & 768 & $100.0 \%$ & $96.1 \%$ & $96.2 \%$ & 0.522 \\
\hline HPV51 & 9 & 33 & 0 & 775 & $100.0 \%$ & $95.9 \%$ & $96.0 \%$ & 0.341 \\
\hline HPV39 & 14 & 17 & 1 & 785 & $93.3 \%$ & $97.9 \%$ & $97.8 \%$ & 0.599 \\
\hline HPV6 & 51 & 22 & 3 & 741 & $94.4 \%$ & $97.1 \%$ & $96.9 \%$ & 0.787 \\
\hline HPV11 & 21 & 13 & 3 & 780 & $87.5 \%$ & $98.4 \%$ & $98.0 \%$ & 0.714 \\
\hline HPV81 & 29 & 22 & 4 & 762 & $87.9 \%$ & $97.2 \%$ & $96.8 \%$ & 0.675 \\
\hline Total & 885 & 405 & 100 & 13316 & $89.8 \%$ & $97.0 \%$ & $96.6 \%$ & 0.760 \\
\hline
\end{tabular}

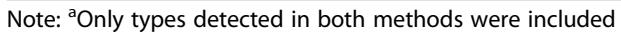

detected by the BMRT HPV PCR assay than those detected by the HPV GenoArray test.

\section{Accuracy of the BMRT HPV PCR assay compared with sequencing results}

As shown in Table 2, the sensitivity, specificity and concordance rate (accuracy) of the BMRT HPV PCR assay was $98.4 \%, 99.6 \%$ and $99.6 \%$ by comparing with sequencing results, respectively. For detection of individual HPV types, $100 \%$ sensitivity was achieved by the method for detections of $13 \mathrm{HPV}$ genotypes, including HPV-59, -66, $-53,-45,-56,-35,-68,-51,-39,-82,-26,-73$ and -11 , and $100 \%$ specificity and accuracy for HPV-66, $-45,-82$ and -73 . The accuracies for detections of HPV-18, -58 and -52 by BMRT HPV PCR assay were $99.4 \%$, $99.4 \%$ and $99.1 \%$, respectively. However, the accuracy for detection of HPV-16, the most common HPV type, was the lowest one (98.8\%), even though it had the highest number of samples compared to the other HPV types. Compared with the sequencing results, the overall PPV and NPV of the BMRT HPV PCR assay was $95.7 \%$ and $99.9 \%$, respectively. Identical with the specificity and sensitivity, the PPV of the BMRT HPV PCR assay for detections of HPV-66, $-45,-82$ and -73 were $100 \%$, and the NPV for detections of HPV-59, -66, -53, -45, -56, -35, $68,51,39,82,26,73$ and 11 were $100 \%$.
Compared with sequencing results, the HPV genotyping by the BMRT HPV PCR assay showed perfect agreement $(k=0.968 ; 95 \% C I, 0.961-0.975)$; Meanwhile, the accuracy of the BMRT HPV PCR assay was $91.6 \%$ (95\% $\mathrm{CI}, 88.8-94.0, \mathrm{n}=467)$ and $90.8 \%(95 \% \mathrm{CI}, 87.3-93.7$, $\mathrm{n}=350$ ) for samples with single and multiple infections, respectively. For detections of individual HPV types, the BMRT HPV PCR assay and sequencing showed almost perfect agreement.

\section{Analyses of discordant HPV typing results between the BMRT HPV PCR assay and sequencing by comparing to the historical diagnoses and infection states of the cases} The discordant HPV typing results between the BMRT HPV PCR assay and sequencing were estimated for single and multiple infections (Table 3). For detections of all HPV types, total 76 cases showed discordant in BMRT HPV PCR assay and sequencing results. Among the 76 discordant cases, 20 cases (6 cases of single infection and 14 of multiple infections) were detected by the sequencing but not by the BMRT HPV PCR assay. In contrast, the other 56 cases (32 cases of single infection and 24 of multiple infections) were identified by the BMRT HPV PCR assay but not by the sequencing.

Among the 65 multiple infected cases, which determined by either the BMRT HPV PCR assay or sequencing, 51 cases were positive for at least one additional 
Table 2 Accuracy of the BMRT HPV PCR assay compared with sequencing from all the 817 samples

\begin{tabular}{|c|c|c|c|c|c|c|c|c|c|c|c|c|c|}
\hline Type & $++^{\mathrm{a}} /+^{\mathrm{b}}$ & $+/-$ & $-/+$ & $-/-$ & Sensitivity & Specificity & $\begin{array}{l}\text { False positive } \\
\text { rate }\end{array}$ & $\begin{array}{l}\text { False negative } \\
\text { rate }\end{array}$ & $\begin{array}{l}\text { Concordance } \\
\text { rate }\end{array}$ & PPV & NPV & k & $95 \% \mathrm{Cl}$ \\
\hline HPV16 & 361 & 7 & 3 & 446 & $99.2 \%$ & $98.5 \%$ & $1.5 \%$ & $0.8 \%$ & $98.8 \%$ & $98.1 \%$ & $99.3 \%$ & 0.975 & $0.960-0.991$ \\
\hline HPV18 & 138 & 1 & 4 & 674 & $97.2 \%$ & $99.9 \%$ & $0.1 \%$ & $2.8 \%$ & $99.4 \%$ & $99.3 \%$ & $99.4 \%$ & 0.979 & $0.960-0.997$ \\
\hline HPV31 & 35 & 1 & 1 & 780 & $97.2 \%$ & $99.9 \%$ & $0.1 \%$ & $2.8 \%$ & $99.8 \%$ & $97.2 \%$ & $99.9 \%$ & 0.971 & $0.931-1.011$ \\
\hline HPV59 & 32 & 3 & 0 & 782 & $100.0 \%$ & $99.6 \%$ & $0.4 \%$ & $0.0 \%$ & $99.6 \%$ & $91.4 \%$ & $100.0 \%$ & 0.953 & $0.901-1.006$ \\
\hline HPV66 & 33 & 0 & 0 & 784 & $100.0 \%$ & $100.0 \%$ & $0.0 \%$ & $0.0 \%$ & $100.0 \%$ & $100.0 \%$ & $100.0 \%$ & 1 & $\sim$ \\
\hline HPV53 & 54 & 3 & 0 & 760 & $100.0 \%$ & $99.6 \%$ & $0.4 \%$ & $0.0 \%$ & $99.6 \%$ & $94.7 \%$ & $100.0 \%$ & 0.971 & $0.938-1.004$ \\
\hline HPV33 & 39 & 1 & 3 & 774 & $92.9 \%$ & $99.9 \%$ & $0.1 \%$ & $7.1 \%$ & $99.5 \%$ & $97.5 \%$ & $99.6 \%$ & 0.949 & $0.896-0.999$ \\
\hline HPV58 & 85 & 1 & 4 & 727 & $95.5 \%$ & $99.9 \%$ & $0.1 \%$ & $4.5 \%$ & $99.4 \%$ & $98.8 \%$ & $99.5 \%$ & 0.968 & $0.940-0.996$ \\
\hline HPV45 & 20 & 0 & 0 & 797 & $100.0 \%$ & $100.0 \%$ & $0.0 \%$ & $0.0 \%$ & $100.0 \%$ & $100.0 \%$ & $100.0 \%$ & 1 & $\sim$ \\
\hline HPV56 & 56 & 9 & 0 & 752 & $100.0 \%$ & $98.8 \%$ & $1.2 \%$ & $0.0 \%$ & $98.9 \%$ & $86.2 \%$ & $100.0 \%$ & 0.920 & $0.868-0.972$ \\
\hline HPV52 & 104 & 5 & 2 & 706 & $98.1 \%$ & $99.3 \%$ & $0.7 \%$ & $1.9 \%$ & $99.1 \%$ & $95.4 \%$ & $99.7 \%$ & 0.963 & $0.935-0.990$ \\
\hline HPV35 & 21 & 3 & 0 & 793 & $100.0 \%$ & $99.6 \%$ & $0.4 \%$ & $0.0 \%$ & $99.6 \%$ & $87.5 \%$ & $100.0 \%$ & 0.932 & $0.854-1.008$ \\
\hline HPV68 & 48 & 1 & 0 & 768 & $100.0 \%$ & $99.9 \%$ & $0.1 \%$ & $0.0 \%$ & $99.9 \%$ & $98.0 \%$ & $100.0 \%$ & 0.989 & $0.968-1.011$ \\
\hline HPV51 & 40 & 2 & 0 & 775 & $100.0 \%$ & $99.7 \%$ & $0.3 \%$ & $0.0 \%$ & $99.8 \%$ & $95.2 \%$ & $100.0 \%$ & 0.974 & 0.939-1.010 \\
\hline HPV39 & 30 & 1 & 0 & 786 & $100.0 \%$ & $99.9 \%$ & $0.1 \%$ & $0.0 \%$ & $99.9 \%$ & $96.8 \%$ & $100.0 \%$ & 0.983 & $0.950-1.016$ \\
\hline HPV82 & 12 & 0 & 0 & 805 & $100.0 \%$ & $100.0 \%$ & $0.0 \%$ & $0.0 \%$ & $100.0 \%$ & $100.0 \%$ & $100.0 \%$ & 1 & $\sim$ \\
\hline HPV26 & 2 & 1 & 0 & 814 & $100.0 \%$ & $99.9 \%$ & $0.1 \%$ & $0.0 \%$ & $99.9 \%$ & $66.7 \%$ & $100.0 \%$ & 0.799 & $0.415-1.184$ \\
\hline HPV73 & 4 & 0 & 0 & 813 & $100.0 \%$ & $100.0 \%$ & $0.0 \%$ & $0.0 \%$ & $100.0 \%$ & $100.0 \%$ & $100.0 \%$ & 1 & $\sim$ \\
\hline HPV6 & 70 & 3 & 1 & 743 & $98.6 \%$ & $99.6 \%$ & $0.4 \%$ & $1.4 \%$ & $99.5 \%$ & $95.9 \%$ & $99.9 \%$ & 0.970 & $0.940-0.999$ \\
\hline HPV11 & 26 & 8 & 0 & 783 & $100.0 \%$ & $99.0 \%$ & $1.0 \%$ & $0.0 \%$ & $99.0 \%$ & $76.5 \%$ & $100.0 \%$ & 0.862 & $0.767-0.956$ \\
\hline HPV81 & 45 & 6 & 2 & 764 & $95.7 \%$ & $99.2 \%$ & $0.8 \%$ & $4.3 \%$ & $99.0 \%$ & $88.2 \%$ & $99.7 \%$ & 0.913 & $0.854-0.973$ \\
\hline Total & 1255 & 56 & 20 & 15826 & $98.4 \%$ & $99.6 \%$ & $0.4 \%$ & $1.6 \%$ & $99.6 \%$ & $95.7 \%$ & $99.9 \%$ & 0.968 & $0.961-0.975$ \\
\hline
\end{tabular}

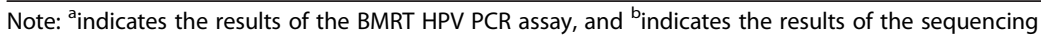

HPV type by the BMRT HPV PCR assay than sequencing. While, among the 23 discordant cases of patients with CINs and cancer, 19 cases were detected by the BMRT HPV PCR assay, but only 4 cases with CINs were detected by sequencing. These results indicate that the BMRT HPV PCR assay may be more suitable for detecting multiple infections in women with pathological lesions than sequencing method.

\section{Relationship between relative DNA loads of HPV-16 or} HPV-18 and histomorphological findings of infected women All cervical scrapes from HPV-16 and HPV-18 positive women were taken in the course of a colposcopic examination and biopsies. Altogether, 313 HPV-16 and 130 HPV18 positive patients fulfilled this criterion. Among the 313 HPV-16 positive patients, 171 were NILM/cervicitis, 56 were LSIL/ASCUS/ASC-H/CINI, 63 were HSIL/CINII-III and 23 were cervical cancer. The relationships between relative loads of HPV-16 DNA (copies per 10,000 cells) in corresponding cervical scrapes and the histopathological findings of the patients were analyzed. As shown in Fig. 1, the median viral load (lg) in patients with NILM/cervicitis, LSIL/ASCUS/ASC-H/CINI, HSIL/CINII-III and cancer were 3.33, 4.75, 4.99 and 5.13, respectively. Moreover, pairwise comparisons among the four groups by KruskalWallis test showed no significant differences of viral loads in samples between the two CIN groups, as well as between each of the two CIN groups and the cancer group. However, highly marked differences were observed when comparing the group presenting NILM/cervicitis to either all grades of CIN $(p<0.001)$, or to cervical carcinomas group $(p<0.001)$ by Mann-Whitney $U$ test. As shown in Fig. 2, the median viral loads (copies per 10,000 cells) in patients with NILM/cervicitis and ASCUS/CINI-III/cancer were 3.33 and 4.97, respectively.

To discriminate the group of NILM and cervicitis from CINI-III and cancer, the area under the curve (AUC) for HPV-16 viral load was calculated for two endpoints of CINI and greater, and of CINII and greater. For HPV-16, the AUCs were 0.827 for CINI and greater, and 0.786 for CINII and greater. The optimal cutoff value of 16,600 copies per 10,000 cells was selected. This value corresponds to $74.6 \%$ sensitivity and $72.5 \%$ specificity for predicting CINI and greater, to $80.2 \%$ sensitivity and $63.0 \%$ specificity for predicting CINII and greater. 
Table 3 Analyses of discordant HPV typing results between the BMRT PCR assay and sequencing by comparing to the historical diagnoses and infection states of the cases

\begin{tabular}{|c|c|c|c|c|c|c|}
\hline \multirow[t]{3}{*}{ Type } & \multicolumn{3}{|c|}{ Numbers of BMRT negative/sequencing positive } & \multicolumn{3}{|c|}{ Numbers of BMRT positive/sequencing negative } \\
\hline & \multicolumn{2}{|c|}{ Infection states } & \multirow{2}{*}{ 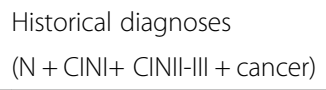 } & \multicolumn{2}{|c|}{ Infection states } & \multirow{2}{*}{$\begin{array}{l}\text { Historical diagnoses } \\
(\mathrm{N}+\mathrm{CINI}+\mathrm{CINII}-\mathrm{III}+\text { cance }\end{array}$} \\
\hline & $\overline{T o t a l}$ & $(\text { Single }+ \text { multiple })^{a}$ & & $\overline{\text { Total }}$ & $\left(\right.$ Single + multiple $\left.{ }^{a}\right)$ & \\
\hline HPV16 & 3 & $(2+1)$ & $(3+0+0+0)$ & 7 & $(0+7)$ & $(7+0+0+0)$ \\
\hline HPV18 & 4 & $(0+4)$ & $(1+2+1+0)$ & 1 & $(0+1)$ & $(1+0+0+0)$ \\
\hline HPV31 & 1 & $(1+0)$ & $(0+0+1+0)$ & 1 & $(0+1)$ & $(1+0+0+0)$ \\
\hline HPV59 & 0 & & & 3 & $(1+2)$ & $(2+0+1+0)$ \\
\hline HPV53 & 0 & & & 3 & $(0+3)$ & $(0+3+0+0)$ \\
\hline HPV33 & 3 & $(1+2)$ & $(3+0+0+0)$ & 1 & $(0+1)$ & $(1+0+0+0)$ \\
\hline HPV58 & 4 & $(0+4)$ & $(4+0+0+0)$ & 1 & $(0+1)$ & $(1+0+0+0)$ \\
\hline HPV56 & 0 & & & 9 & $(1+8)$ & $(7+0+1+1)$ \\
\hline HPV52 & 2 & $(1+1)$ & $(2+0+0+0)$ & 5 & $(1+4)$ & $(3+0+1+1)$ \\
\hline HPV35 & 0 & & & 3 & $(0+3)$ & $(0+0+2+1)$ \\
\hline HPV68 & 0 & & & 1 & $(1+0)$ & $(1+0+0+0)$ \\
\hline HPV51 & 0 & & & 2 & $(0+2)$ & $(1+0+1+0)$ \\
\hline HPV39 & 0 & & & 1 & $(0+1)$ & $(1+0+0+0)$ \\
\hline HPV26 & 0 & & & 1 & $(0+1)$ & $(1+0+0+0)$ \\
\hline HPV6 & 1 & $(0+1)$ & $(1+0+0+0)$ & 3 & $(0+3)$ & $(1+1+1+0)$ \\
\hline HPV11 & 0 & & & 8 & $(1+7)$ & $(3+1+4+0)$ \\
\hline HPV81 & 2 & $(1+1)$ & $(2+0+0+0)$ & 6 & $(0+6)$ & $(6+0+0+0)$ \\
\hline Total & 20 & $(6+14)$ & $(16+2+2+0)$ & 56 & $(5+51)$ & $(37+5+11+3)$ \\
\hline
\end{tabular}

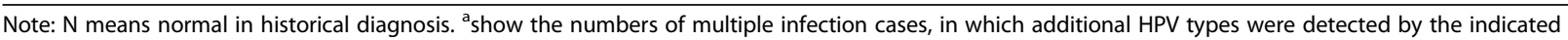
(Positive) method

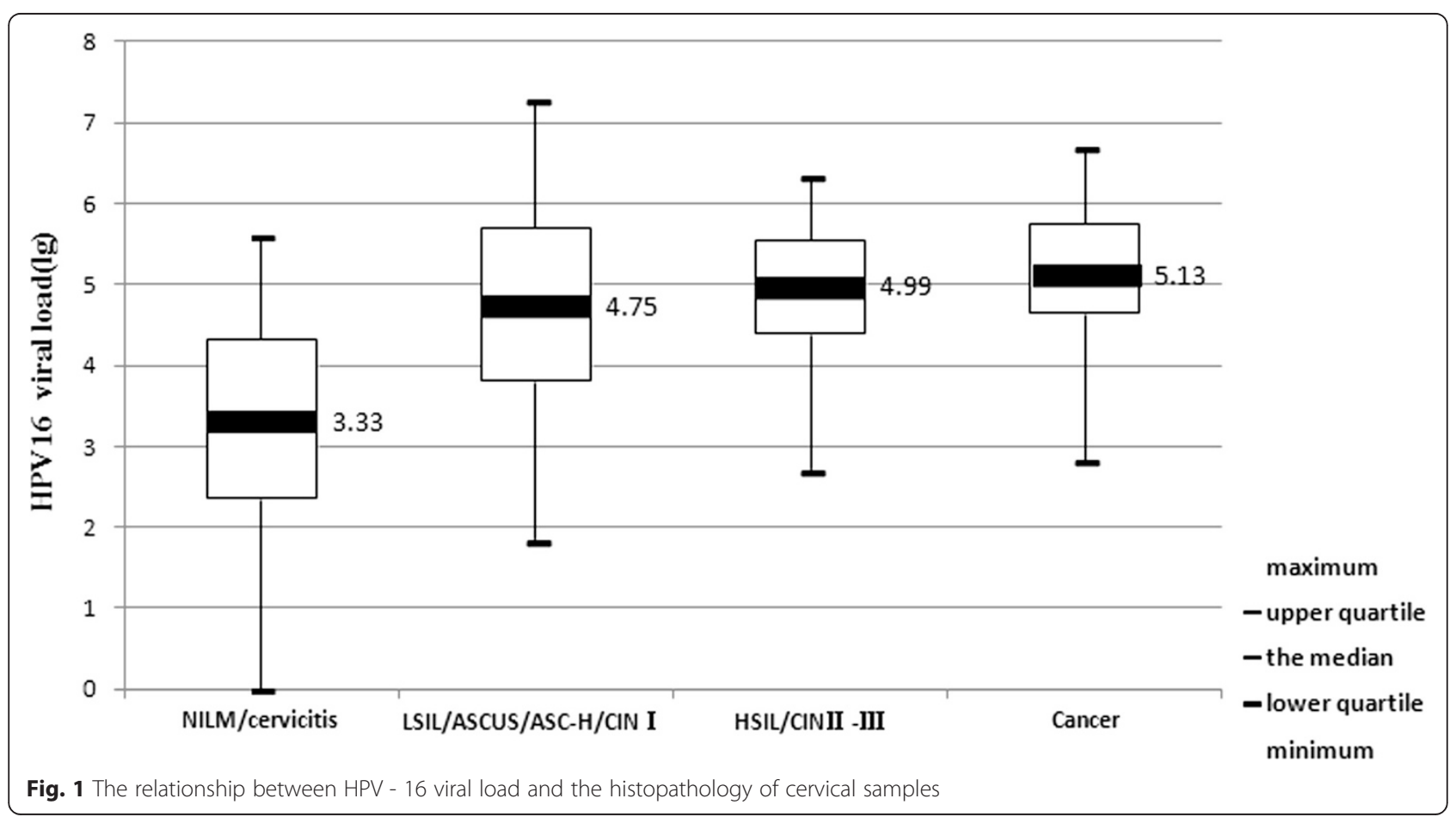




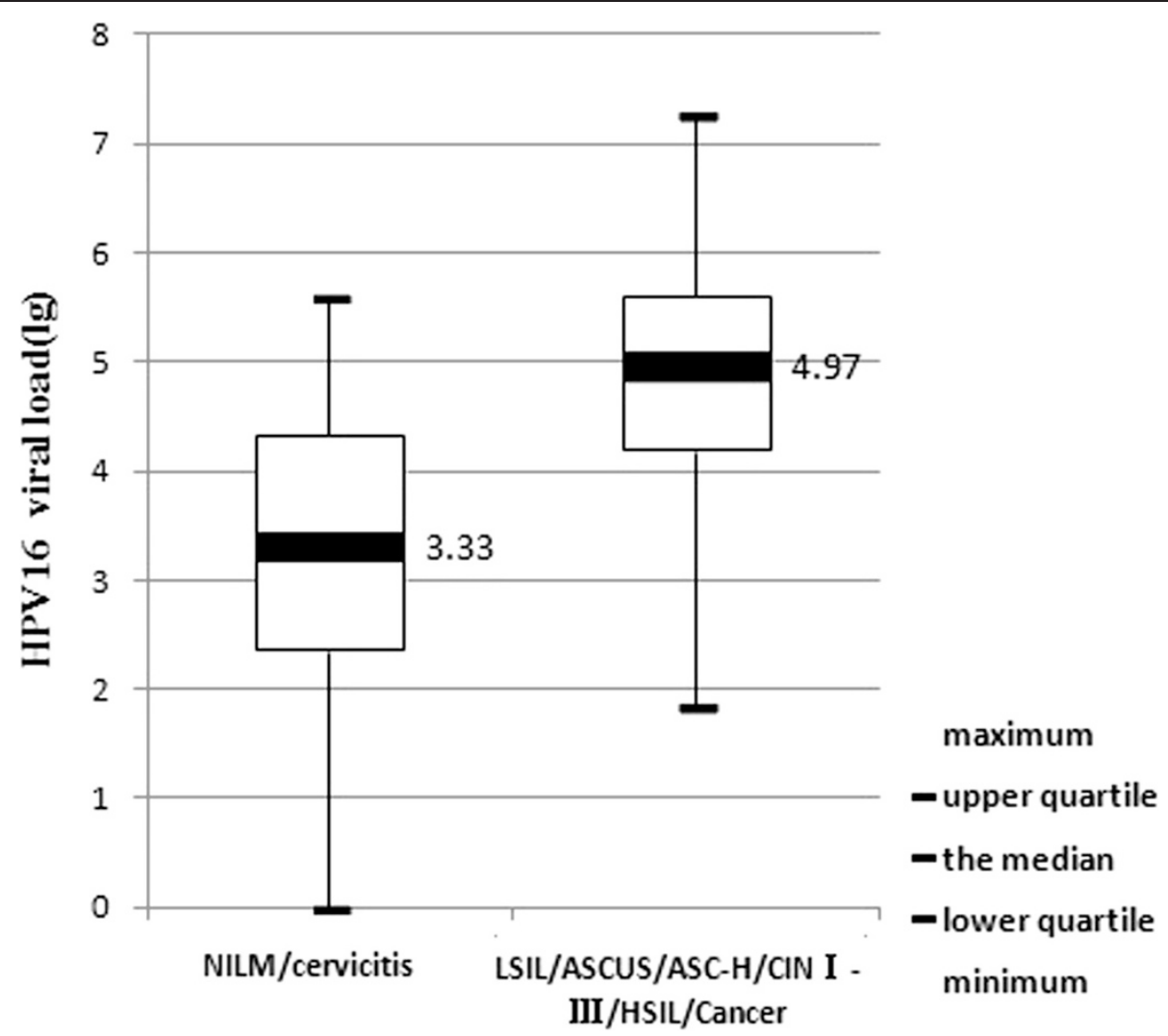

Fig. 2 Comparison of HPV - 16 viral loads between NILM/cervicitis and ASCUS/CIN I-III/cancer

Among $130 \mathrm{HPV}-18$ positive patients, 81 were negative in histological diagnosis (including normal, infusorian and cervicitis), 27 were LSIL/ASCUS/ASC-H/CINI, 14 were HSIL/CINII-III, and 8 were cervical cancer. The relative levels of HPV-18 DNA loads (copies per 10,000 cells) in corresponding cervical scrapes were calculated to correlate them with the histopathological findings of infected patients (Fig. 3). The median viral load (lg) in patients with diagnosis of NILM/cervicitis, LSIL/ASCUS/ ASC-H/CINI, HSIL/CINII-III and cancer were 3.21, 4.84, 3.83 and 3.92, respectively. Moreover, pairwise comparisons among the four groups by Kruskal-Wallis test showed highly significant difference of viral loads in samples between the group presenting NILM/cervicitis and the group of LSIL/ASCUS/ASC-H/CINI $(p<0.001)$. Unlike those of HPV16, the viral loads of HPV-18 were less associated with progress of CINII-III. Similar to those of HPV-16, the significant differences of HPV-18 viral loads were observed when comparing the group presenting NILM/cervicitis to either all grades of CIN $(p<0.001)$, or to cervical carcinomas group $(p<0.001)$ by Mann-Whitney $U$ test. As shown in Fig. 4, the median viral loads (copies per 10,000 cells) in patients with NILM/cervicitis and ASCUS/CINI-III/cancer were 3.21 and 4.31, respectively.

\section{Discussion}

In clinical screening of HPV-infected women, accurate HPV genotyping has become an important prognostic indicator for monitoring persistent HPV infection, which is the strong causality of high grade cervical intraepithelial neoplasia $[18,20]$. In our hospital, HPV DNA is routinely detected in Cervical Cancer Screening Program by the HPV GenoArray method, which allows for genotyping of $13 \mathrm{HR}$ types, 5 LR types and 3 other types commonly found in China [18]. However, the HPV GenoArray method does not provide quantitative information on detected HPV DNA. Evidence is accumulating that HPV quantification may be useful as genotyping for patient management in the future. In order to solve this problem, a multiplex real time assay was designed for simultaneous genotyping and quantification of the 18 most frequent cancer related HRHPV types and 3 LR-HPV types of HPV-6, -11 and -81 . A recent cross-sectional study showed that these types mentioned above were the predominant HPV types in women with high-grade lesions [21].

Quantitative real-time PCR methods are considered to be the gold standard for HPV load assessment, but these have not been developed and validated for the genotyping and quantifying wide spectrum of carcinogenic HPV types often encountered in cervical samples [22] . HPV 


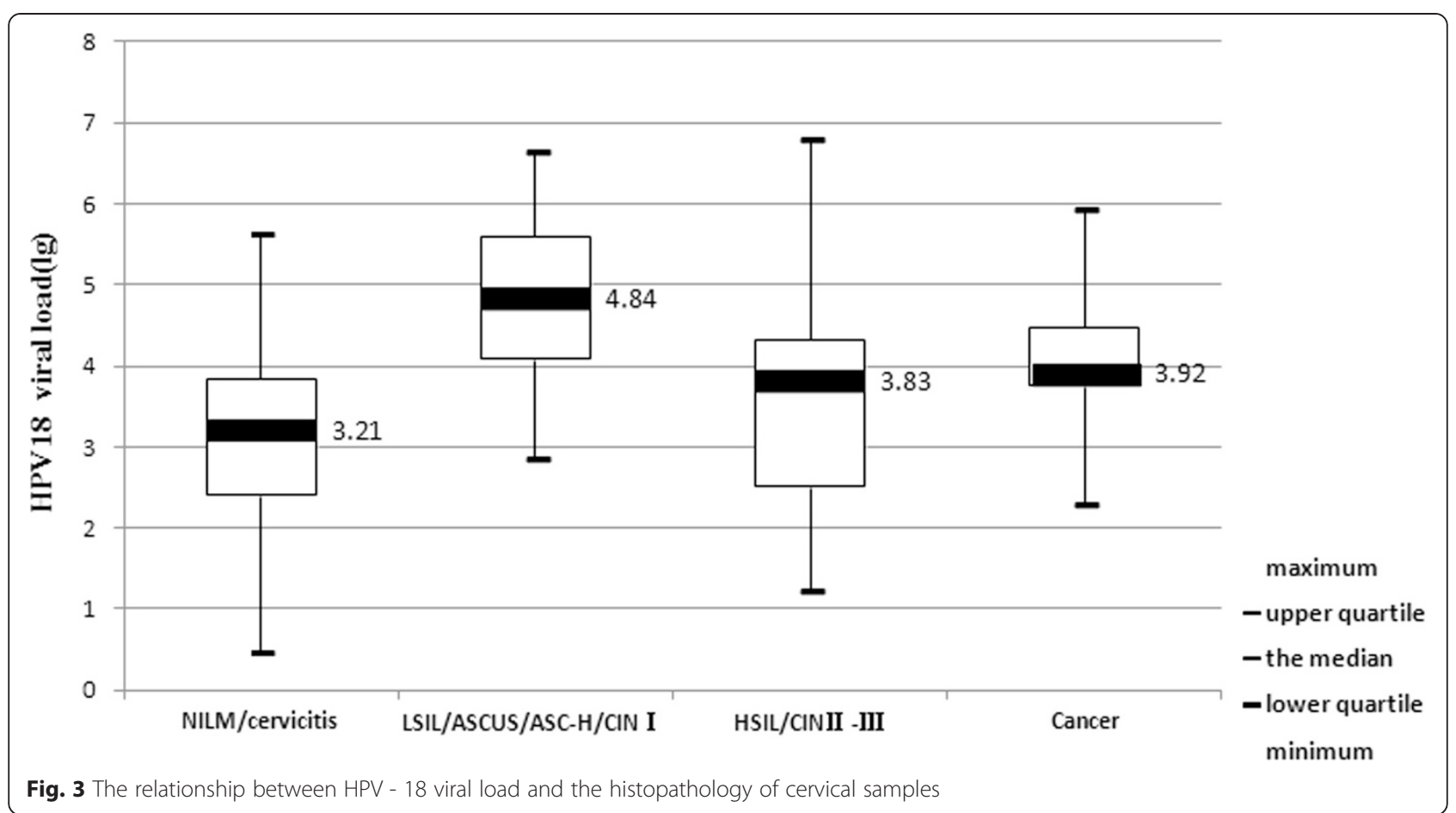

Fig. 3 The relationship between HPV - 18 viral load and the histopathology of cervical samples

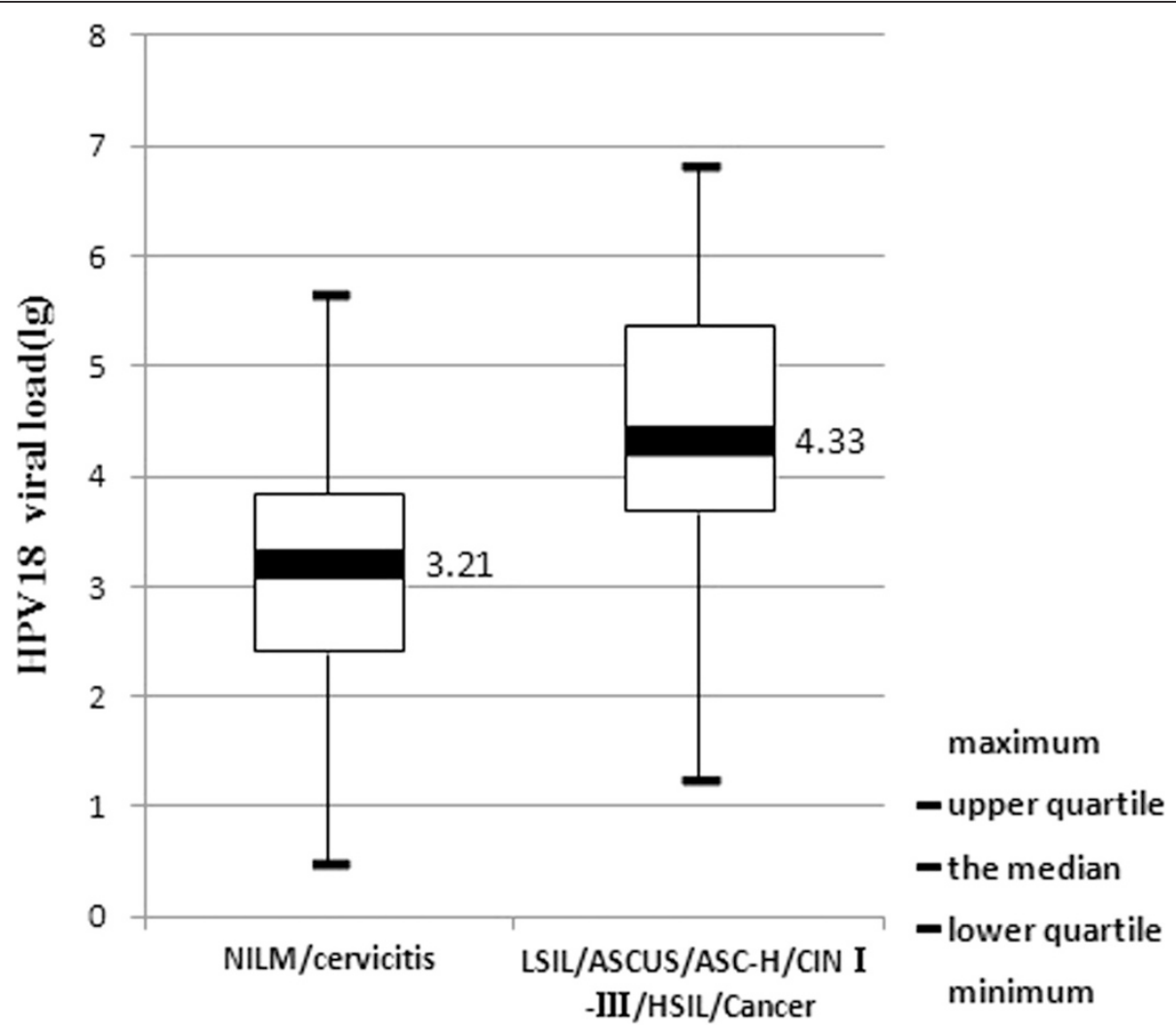

Fig. 4 Comparsion of HPV - 18 viral loads between NILM/cervicitis and ASCUS/CIN I-III/cancer 
DNA viral load, usually estimated as the amount of HPV genome copies per cell, has been variably associated with cervical disease, and appears to have an overall specificity to differentiate normal cytology from abnormal cytology [23]. A novel application of the BMRT HPV PCR assay for the HPV genotyping and quantifying is described. The BMRT HPV PCR assay is a multiplex gene analysis platform, offers a high sensitive, cost-effectiveness and high throughput assay that allows the rapid and specific detection of 21 high-risk and low-risk HPV genotypes in conjunction with BMRT genetic analysis system. Each pair of HPV-specific primers only generated a single peak for each HPV genotype in addition to the internal control peak. Analyses of 817 specimens using the BMRT PCR assay and the HPV GenoArray test demonstrated that the BMRT PCR assay had comparable sensitivity and specificity to HPV GenoArray test (Table 2). In this study, a $96.6 \%$ of total coincidence rate and a substantial almost perfect agreement $(k=0.760)$ for genotyping 18 HPV types, which are capable detected in both the methods, was achieved between the BMRT HPV PCR assay and the HPV GenoArray test. The BMRT HPV PCR assay was proved to be highly specific for typing the $21 \mathrm{HPV}$ types. This is a particular note since both assays differ considerably in their design. The HPV GenoArray test is based on amplification by a pair of consensus primers in a highly conserved region of the L1 gene and reverse hybridization to the type-specific probes, whereas the BMRT HPV PCR assay utilizes type-specific primers and TaqMan probes to amplify the sequences within the L1 region. Numerous studies have been showed that the GenoArray test is a highly reproducible assay with an excellent clinical sensitivity for HPV types and is thus considered to be adequate for comparison $[18,20]$. Most of the discordant results detected by the two methods were seen in cases with multiple infections. Generally, agreement is relatively poor between various assays for genotyping multiple HPV infections. A recent study, which evaluated different multiplex HPV PCR assays for identification of low prevalent HPV types, revealed only moderate inter-assay agreement in cases of single HPV infections and poor agreement in cases with multiple HPV infections [24].

DNA sequencing is the "gold standard" method for accurate HPV genotyping, in which HPV DNA is amplified with specific primers followed by sequencing. It is desirable to evaluate the accuracy of any HPV genotyping method on the basis of the sequencing results $[25,26]$. In this study, a perfect agreement $(k=0.968 ; 95 \% \mathrm{CI}, 0.961-0.975)$ for HPV genotyping between the BMRT HPV PCR assay and sequencing was obtained. Compared with sequencing method, the sensitivity, specificity and accuracy of the BMRT HPV PCR assay was 98.4 \%, $99.6 \%$ and $99.6 \%$, respectively. This result indicates that HPV genotype could be successfully identified by the BMRT HPV PCR assay with high accuracy.

The BMRT HPV PCR assay was demonstrated to have good sensitivity, specificity, accuracy, PPV and NPV compared to the sequencing method. Among detections of the $21 \mathrm{HPV}$ genotypes, detections of $13 \mathrm{HPV}$ genotypes by the BMRT HPV PCR assay showed $100 \%$ sensitivity and NPV value, detections of 4 HPV genotypes showed $100 \%$ specificity and PPV value. All HPV infections in patients with CINs and cancer were successfully detected by the BMRT HPV PCR assay except for 2 cases of patients with CINII-III and 2 with CIN. Of the $817 \mathrm{HPV}$ positive samples (Detections of the $21 \mathrm{HPV}$ genotypes were performed in each sample), only 20 cases were positive (in 6 single infection cases and for additional types in 14 multiple infection cases) in the sequencing test but negative in the BMRT HPV PCR assay. However, among these 20 cases only two were diagnosed as CINII-III and the others were diagnosed as normal, cervicitis and CINI. This result suggests that the BMRT PCR method could successfully detect HPV infections and identify HPV genotypes with a high degree of accuracy.

In this study, the results showed that the median relative levels of HPV-16 DNA do not vary by more than 2folds irrespective of the severity of CIN. There is also no statistical significance of viral loads in samples between the two CIN groups, as well as between each of the two CIN groups and the cancer group by Kruskal-Wallis test. The data indicate that viral load does not correlate with disease progression within the CIN spectrum. However, highly significant differences of viral loads were achieved when comparing the group lacking CIN with the group comprising of all CIN $(p<0.001)$ or with the cancer group $(p<0.001)$. A significant decrease of viral load in liquid based cytology samples from cervical carcinoma patients compared to those from CIN patients was reported by Yoshida et al [27]. However, our quantitative data showed that the relative number of HPV genomic copies within a histological entity can vary within the upper and lower quartile of the box plot (Fig. 1). The differences are several log-folds when considering the extreme values of each group. Similar observations were reported in a recent study in which the variation in HPV-16 viral load within different histological grades of cervical neoplasia were evaluated [28]. It is therefore likely that changes in viral load over time, rather than a single measurement, might be predictive for disease progression or clearance [17].

In contrast to HPV-16, viral loads of HPV-18 were less associated with CINII-III or cancer. This observation may be due to the different viral activity between HPV-18 and HPV-16, or a higher proportion of HPV-18 infections in 
patients with glandular lesions that are more difficult to sample and hence are prone to false-negative results. Our data suggest that careful attention should be paid to HPV18 as the same as to HPV-16, but for a different reason. Specifically, the possible use of HPV-18 typing to improve the detection of cytologically occult lesions should be formally evaluated. Extended analyses, accounting for multiple carcinogenic infections, are necessary to evaluate the role of viral load for other carcinogenic HPV types.

From an economic point, the BMRT HPV PCR assay costs less and is faster (less than $2.5 \mathrm{~h}$, including DNA extraction). Moreover, the real-time PCR assay enables reliable quantifications of the target DNA. Our approach provides a potential for viral load assessment for 21 types in parallel (not only HPV-16 and HPV-18). Additionally, the assay will be useful to evaluate the clinical relevance of viral persistence at the genotype level, monitor disease recurrence, and examine the effects of widespread vaccination on prevalent HPV types in the future.

\section{Conclusion}

The BMRT assay showed a similar clinical performance for genotyping compared with the HPV GenoArray test and seemed to be a good alternative approach for HRHPV testing,due to its high level of automation and ability to quantify HPV - 16, HPV - 18 and other HR-HPV. Moreover, the BMRT assay could potentially promote patient management by risk stratification of cytological abnormal populations. This new assay could be a useful tool for both primary screening of cervical cancer and the triage of women with abnormal cytology.

\begin{abstract}
Abbreviations
HPV: Human papillomavirus; NILM: Intraepithelial lesion or malignancy; BMRT: BioPerfectus Multiplex Real Time; CIN: Cervical intraepithelial neoplasia grade; RLB: Reverse line blot; LR: Low risk; GAPDH: Glyceraldehyde 3-phosphate dehydrogenase; UDG: Uracil-DNA-Glycosylase; ASCUS: Atypical squamous cells of undetermined significance; LSIL: Low-grade squamous intraepithelial lesions; HSIL: High grade squamous intraepithelial lesions; AUC: Area under the curve.
\end{abstract}

\section{Competing interests}

The present test is the subject of Chinese patent [ZL201110087602.1], and dependent patent applications worldwide held by Jiangsu Bioperfectus Technologies Limited company, of which Xu Zhang is sole shareholders, Rong Zhang, Zhonghua Liu and Xiulin Li are co-inventors.

\section{Authors' contribution}

ZS, RZ and ZL carried out the BioPerfectus multiplex real time, statistical analysis. and drafted the manuscript. $\mathrm{CL}, \mathrm{XL}, \mathrm{WZ}$ and $\mathrm{LY}$ performed the clinical detection. QR and XZ conceived the study, and participated in its design and coordination and helped to draft the manuscript. All authors read and approved the final manuscript.

\section{Acknowledgments}

This work was supported by the National Natural Science Foundation of China (81171580 and 81171581) and the Outstanding Scientific Fund of Shengjing Hospital.

Received: 8 May 2015 Accepted: 30 October 2015

Published online: 06 November 2015

\section{References}

1. Rao A, Young S, Erlich H, Boyle S, Krevolin M, Sun R, et al. Development and characterization of the cobas human papillomavirus test. J Clin Microbiol. 2013:51:1478-84

2. Bouvard V, Baan R, Straif K, Grosse Y, Secretan B, El Ghissassi F, et al. A review of human carcinogens-Part B: biological agents. Lancet Oncol. 2009;10:321-2.

3. Tabrizi SN, Brotherton JM, Stevens MP, Condon JR, Mclntyre P, Smith D, et al. HPV genotype prevalence in Australian women undergoing routine cervical screening by cytology status prior to implementation of an HPV vaccination program. J Clin Virol. 2014;60:250-6.

4. Ong J, Read TR, Vodstrcil LA, Walker S, Chen M, Bradshaw CS, et al. Detection of oral human papillomavirus in HIV-positive men who have sex with men 3 years after baseline: a follow up cross-sectional study. PLoS One. 2014;9:e102138.

5. Li N, Franceschi S, Howell-Jones R, Snijders PJ, Clifford GM. Human papillomavirus type distribution in 30,848 invasive cervical cancers worldwide: Variation by geographical region, histological type and year of publication. Int J Cancer. 2011;128:927-35.

6. de Sanjose S, Quint WG, Alemany L, Geraets DT, Klaustermeier JE, Lloveras B, et al. Human papillomavirus genotype attribution in invasive cervical cancer: a retrospective cross-sectional worldwide study. Lancet Oncol. 2010;11:1048-56.

7. Kjaer SK, Frederiksen K, Munk C, Iftner T. Long-term absolute risk of cervica intraepithelial neoplasia grade 3 or worse following human papillomavirus infection: role of persistence. J Natl Cancer Inst. 2010;102:1478-88.

8. Chaturvedi AK, Katki HA, Hildesheim A, Rodriguez AC, Quint W, Schiffman $M$, et al. Human papillomavirus infection with multiple types: pattern of coinfection and risk of cervical disease. J Infect Dis. 2011;203:910-20.

9. Vaccarella S, Franceschi S, Snijders PJ, Herrero R, Meijer CJ, Plummer M. Concurrent infection with multiple human papillomavirus types: pooled analysis of the IARC HPV Prevalence Surveys. Cancer Epidemiol Biomarkers Prev. 2010;19:503-10

10. Chaturvedi AK, Myers L, Hammons AF, Clark RA, Dunlap K, Kissinger PJ, et al. Prevalence and clustering patterns of human papillomavirus genotypes in multiple infections. Cancer Epidemiol Biomarkers Prev. 2005;14:2439-45.

11. Spinillo A, Dal Bello B, Alberizzi P, Cesari S, Gardella B, Roccio M, et al. Clustering patterns of human papillomavirus genotypes in multiple infections. Virus Res. 2009;142:154-9.

12. Mejlhede N, Pedersen BV, Frisch M, Fomsgaard A. Multiple human papilloma virus types in cervical infections: competition or synergy? APMIS 2010:118:346-52.

13. Spinillo A, Dal Bello B, Gardella B, Roccio M, Dacco MD, Silini EM. Multiple human papillomavirus infection and high grade cervical intraepithelial neoplasia among women with cytological diagnosis of atypical squamous cells of undetermined significance or low grade squamous intraepithelial lesions. Gynecol Oncol. 2009;113:115-9.

14. Stoler MH, Wright Jr TC, Sharma A, Apple R, Gutekunst K, et al. High-risk human papillomavirus testing in women with ASC-US cytology: results from the ATHENA HPV study. Am J Clin Pathol. 2011;135:468-75.

15. Lorincz AT, Castle PE, Sherman ME, Scott DR, Glass AG, Wacholder S, et al. Viral load of human papillomavirus and risk of CIN3 or cervical cancer. Lancet. 2002;360:228-9.

16. van Duin M, Snijders PJ, Schrijnemakers HF, Voorhorst FJ, Rozendaal L, Nobbenhuis MA, et al. Human papillomavirus 16 load in normal and abnormal cervical scrapes: an indicator of CIN I//II and viral clearance. Int J Cancer. 2002:98:590-5.

17. Monnier-Benoit S, Dalstein V, Riethmuller D, Lalaoui N, Mougin C, Pretet JL. Dynamics of HPV16 DNA load reflect the natural history of cervical HPVassociated lesions. J Clin Virol. 2006:35:270-7.

18. Sun ZR, Ji YH, Zhou WQ, Zhang SL, Jiang WG, Ruan Q. Characteristics of HPV prevalence among women in Liaoning province, China. Int J Gynaecol Obstet. 2010;109:105-109.

19. Hanai R, Caron PR, Wang JC. Human TOP3: a single-copy gene encoding DNA topoisomerase III. Proc Natl Acad Sci U S A. 1996;93:3653-7.

20. Chen $Q$, Xie LX, Qing ZR, Li LJ, Luo ZY, Lin M, et al. Epidemiologic characterization of human papillomavirus infection in rural Chaozhou, eastern Guangdong Province of China. PLoS One. 2012;7:e32149.

21. Klug SJ, Hukelmann M, Hollwitz B, Duzenli N, Schopp B, et al. Prevalence of human papillomavirus types in women screened by cytology in Germany. J Med Virol. 2007;79:616-25. 
22. Wentzensen N, Gravitt PE, Long R, Schiffman M, Dunn ST, Carreon JD, et al. Human Papillomavirus Load Measured by Linear Array Correlates with Quantitative PCR in Cervical Cytology Specimens. J Clin Microbiol. 2012;50:1564-70

23. Marongiu L, Godi A, Parry JV, Beddows S. Human Papillomavirus 16, 18, 31 and 45 viral load, integration and methylation status stratified by cervical disease stage. BMC Cancer. 2014;14:384.

24. Sabol I, Salakova M, Smahelova J, Pawlita M, Schmitt M, Gasperov NM, et al Evaluation of different techniques for identification of human papillomavirus types of low prevalence. J Clin Microbiol. 2008;46:1606-13.

25. Vernon SD, Unger ER, Williams D. Comparison of human papillomavirus detection and typing by cycle sequencing, line blotting, and hybrid capture. J Clin Microbiol. 2000;38:651-5.

26. Asato T, Maehama T, Nagai Y, Kanazawa K, Uezato H, Kariya K. A large case control study of cervical cancer risk associated with human papillomavirus infection in Japan, by nucleotide sequencing-based genotyping. J Infect Dis. 2004;189:1829-32.

27. Amakura Y, Yoshimura M, Mouri C, Mikage M, Kawahara N, et al. Convenient TLC-based Identification Test for the Crude Drug "Pogostemoni Herba". Yakugaku Zasshi. 2008;128:1833-7.

28. Fiander AN, Hart KW, Hibbitts SJ, Rieck GC, Tristram AJ, Beukenholdt RW, et al. Variation in human papillomavirus type-16 viral load within different histological grades of cervical neoplasia. J Med Virol. 2007;79:1366-9.

\section{Submit your next manuscript to BioMed Central and take full advantage of:}

- Convenient online submission

- Thorough peer review

- No space constraints or color figure charges

- Immediate publication on acceptance

- Inclusion in PubMed, CAS, Scopus and Google Scholar

- Research which is freely available for redistribution 